\title{
Development of Neutron Energy Spectral Signatures for Passive Monitoring of Spent Nuclear Fuels in Dry Cask Storage
}

\author{
Ira Harkness, Ting Zhu, Yinong Liang, Eric Rauch, Andreas Enqvist, and Kelly A. Jordan
}

\begin{abstract}
Demand for spent nuclear fuel dry casks as an interim storage solution has increased globally and the IAEA has expressed a need for robust safeguards and verification technologies for ensuring the continuity of knowledge and the integrity of radioactive materials inside spent fuel casks. Existing research has been focusing on "fingerprinting" casks based on count rate statistics to represent radiation emission signatures. The current research aims to expand to include neutron energy spectral information as part of the fuel characteristics. First, spent fuel composition data are taken from the Next Generation Safeguards Initiative Spent Fuel Libraries, representative for Westinghouse 17x17 PWR assemblies. The ORIGEN-S code then calculates the spontaneous fission and $(\alpha, n)$ emissions for individual fuel rods, followed by detailed MCNP simulations of neutrons transported through the fuel assemblies. A comprehensive database of neutron energy spectral profiles is to be constructed, with different enrichment, burn-up, and cooling time conditions. The end goal is to utilize the computational spent fuel library, predictive algorithm, and a pressurized ${ }^{4} \mathrm{He}$ scintillator to verify the spent fuel assemblies inside a cask. This work identifies neutron spectral signatures that correlate with the cooling time of spent fuel. Both the total and relative contributions from spontaneous fission and $(\alpha, n)$ change noticeably with respect to cooling time, due to the relatively short half-life (18 years) of the major neutron source ${ }^{244} \mathrm{Cm}$. Identification of this and other neutron spectral signatures allows the characterization of spent nuclear fuels in dry cask storage.
\end{abstract}

Index Terms — spent fuel, safeguards, neutron measurement

\section{INTRODUCTION}

$\mathrm{T}$ HE United States currently has commercial spent fuel accumulating at 78 sites in 34 states. This accounts for over 20,000 significant quantities of plutonium in dry cask storage in the United States. The IAEA has expressed a need for robust safeguards and verification technologies for ensuring the continuity of knowledge and integrity of radioactive materials inside spent fuel casks.

The ultimate objective of safeguards is "the timely detection of diversion of significant quantities of nuclear material from peaceful nuclear activities to the manufacture of nuclear

This work was supported in part by the U.S. Department of Energy Nuclear Energy University Programs.

I. Harkness, T. Zhu, Y. Liang, A. Enqvist, and K. A. Jordan are with the Department of Materials Science and Engineering, University of Florida, Gainesville, FL 32611 USA (e-mail: ira@mse.ufl.edu). weapons or of other nuclear explosive devices or for purposes unknown and deterrence of such diversion by the risk of early detection" [1]. This is often achieved using convention safeguards measures while the materials are being transported from reactor, to cooling pool, to dry storage casks. Once the spent fuel is inside a dry storage cask, non-destructive assay (NDA) is more difficult.

Existing research has focused on "fingerprinting" casks based on count rate statistics [2] and radiation signatures [3]. The primary method of cask monitoring uses a Bonner sphere spectrometer. Bonner spheres are advantageous due to the wide energy range measured, however the energy resolution is limited due to the smooth fluence response functions of the thermal neutron detectors [4]. Existing cask monitoring methods are unable to restore continuity of knowledge in cases where existing knowledge has been lost.

The current research aims to expand to include neutron energy spectral information as part of the spent fuel characteristics. Computational resources used include the Next Generation Safeguards Initiative (NGSI) Spent Fuel Library, ORIGEN-S and MCNP6. In this paper, we examine the transported neutron source spectra from individual pins from a Westinghouse 17x17 PWR assembly. This work identifies neutron spectral signatures that correlate with the cooling time of the spent fuel. The goal is to utilize a computational spent fuel library, predictive algorithm, and a pressurized ${ }^{4} \mathrm{He}$ scintillator to verify the spent fuel assemblies inside a cask.

\section{Computational Methodology}

Computational methods in this work include use of the NGSI Spent Fuel Library, ORIGEN-S, and MCNP.

\section{A. Next Generation Safeguards Initiative Spent Fuel Library}

The Next Generation Safeguards Initiative began in 2009 with the goals of quantifying plutonium content in spent nuclear fuel through the use of non-destructive assay (NDA) techniques, assessing the capability of the NDA techniques to verify burnup, cooling time, and initial enrichment, and determining the ability to detect pin diversions in spent fuel

E. Rauch is with Los Alamos National Laboratory, Los Alamos, NM 87545 
assemblies [5]. To achieve these goals, spent fuel assemblies in the form of MCNP input files were developed. NGSI Spent Fuel Library-2a (SFL2a) is a set of 17x17 pressurized water reactor (PWR) fuel assemblies, which were simulated for a broad range of parameters suitable for direct comparison of different NDA techniques [6]. The initial enrichment values used in the library are $2 \%, 3 \%, 4 \%$ and $5 \%$, burnups range from 15 to $60 \mathrm{GWd} / \mathrm{tU}$ incremented by $15 \mathrm{GWd} / \mathrm{tU}$. Cooling times included 1, 5, 20, 40 and 80 years. The NGSI Spent Fuel Library is key to this research as the fuel assembly is modeled pin by pin.

\section{B. ORIGEN-S}

The modern ORIGEN-S code was developed from the ORIGEN (Oak Ridge Isotope GENeration) code in the late 1970s as a depletion and decay analysis code for SCALE (Standardized Computer Analyses for Licensing Evaluation). The primary advantage of ORIGEN-S for spent fuel safeguards is the ability to accurately and quickly predict neutron emissions from spent fuel. The neutron source calculation is based on methods from the SOURCES code, using spontaneous fission Watt spectral parameters for 41 actinides and a matrixdependent $(\alpha, n)$ source method using $(\alpha, n)$ cross sections and yields for $\alpha$ particles on 19 target nuclides, including ${ }^{17} \mathrm{O}$ and ${ }^{18} \mathrm{O}$ which are found in spent fuel. [7]. The ORIGEN-S code and associated nuclear data libraries have been through numerous verification and validation benchmarks. These included validation against more than 100 spent fuel samples from domestic and international programs involving older and modern high-burnup mixed oxide (MOX) and low-enriched uranium (LEU) fuels [8].

\section{C. $M C N P 6$}

MCNP6 is a general-purpose Monte Carlo N-Particle code that can be used to calculate continuous energy, threedimensional, coupled neutron-photon-electron transport. The continuous energy cross section data allow linear interpolation between energy points. MCNP6 includes a general source, criticality source, and surface source along with numerous variance reduction techniques. It also offers a flexible tally structure and an extensive cross-section library. MCNP6 [9] and the ENDF-B/VII library [10] have been validated against numerous transport and shielding scenarios.

\section{Simulations}

The Next Generation Safeguards Initiative (NGSI) Spent Fuel Library was used to obtain nuclide masses from individual rods within spent fuel assemblies of varying initial enrichments, cooling times, and burnups. ORIGEN-S used the nuclide mass data from this rod to calculate the neutron spectra for both spontaneous fission and $(\alpha, n)$ reactions. A MCNP model of a single spent fuel rod with cladding was built using the NGSI spent fuel libraries. Three separate models were built for each rod using the following homogeneous source spectra: spontaneous fission, $(\alpha, n)$ reaction neutrons, and total neutrons. MCNP was used to transport one hundred million neutrons per source spectra with a tally on the outside surface of the cladding.

Due to the large amount of nuclides in each spent fuel assembly along with the number of different assemblies to analyze, a manual method was unfeasible. A methodology was developed to link the NGSI spent fuel assemblies in MCNP input files to ORIGEN-S input files for automatically generating both the spontaneous fission and $(\alpha, n)$ reaction neutron spectra. Further development added the ability to write MCNP input files using the data obtained from the ORIGEN-S output files. The total workflow from NGSI spent fuel library to transported neutron source spectra is shown in Figure 1.

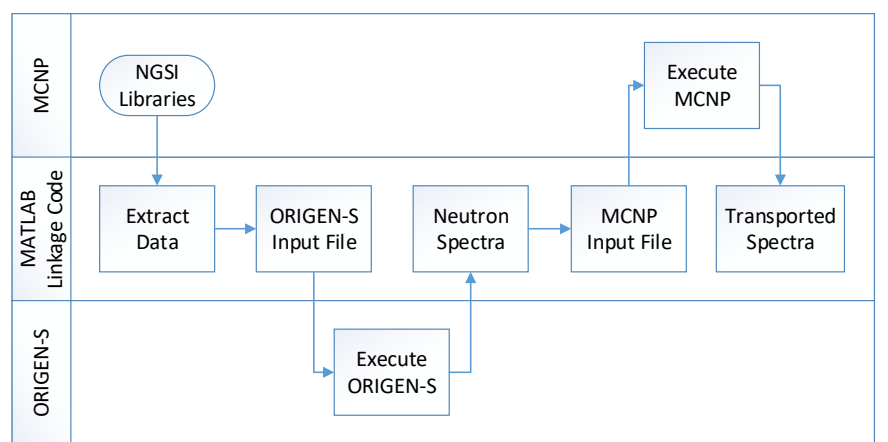

Fig. 1. Total path from NGSI spent fuel library to final transported neutron spectra.

Computational analyses were executed for a single rod from each of the assemblies in the NGSI spent fuel library, which included varying initial enrichments, burnups, and cooling times. The results and discussion below include examination of a rod with an initial enrichment of 4 percent, burnup of 15 GWd/MTU and cooling times of 5, 20, 40, and 80 years.

\section{RESUlTS AND DISCUSSION}

The simulated total neutron flux on the outside of the assembly is shown in Figure 2. There is a decrease in the total neutron flux with increasing cooling time.

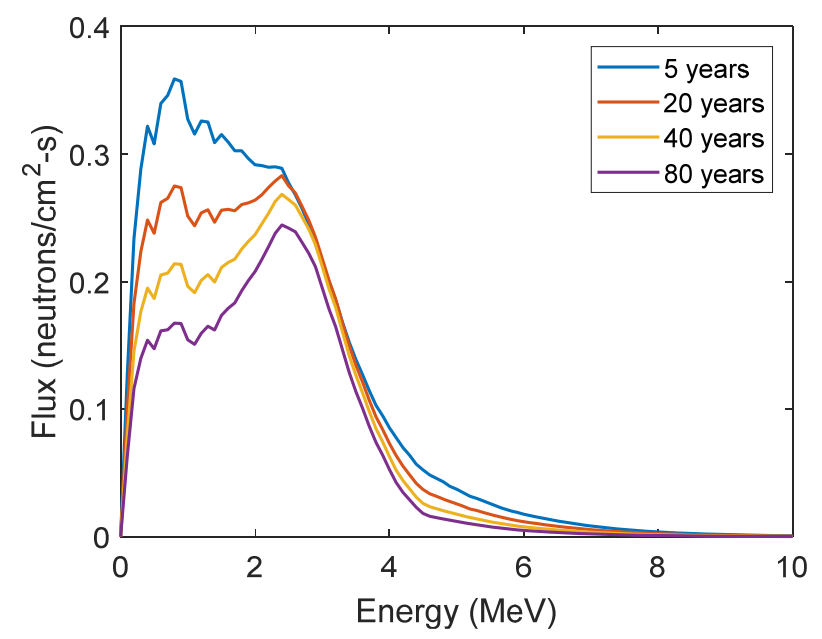

Fig. 2. Change in total neutron spectra as a function of cooling time. The total neutron flux decreases with increasing cooling time, however the peak around 3.0 $\mathrm{MeV}$ persists.

Figure 3 shows the neutron source term fractions separated into total neutron flux, spontaneous fission neutron flux and 
$(\alpha, n)$ reaction neutron flux. The total neutron and spontaneous fission neutron fluxes both decrease, but the neutron flux from the $(\alpha, n)$ reaction increases.

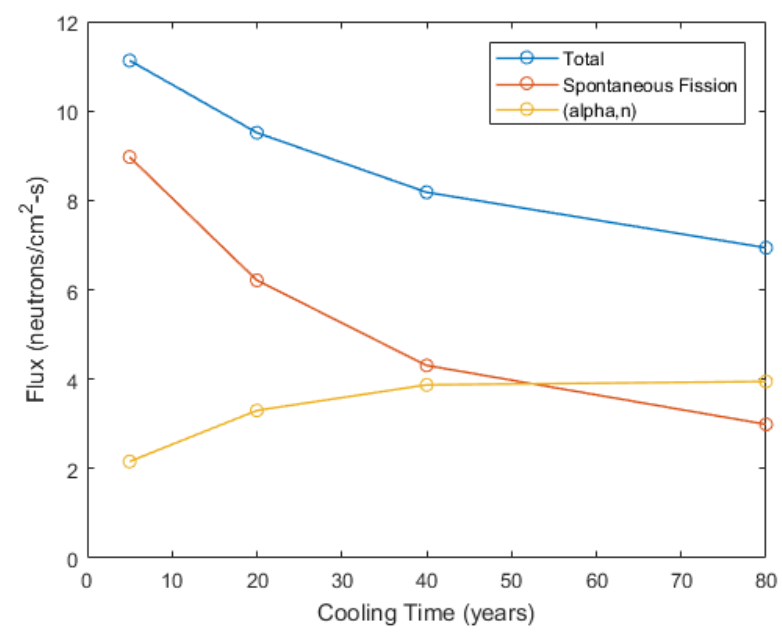

Fig. 3. Change in neutron source strength as a function of cooling time. While the total neutron and spontaneous fission neutron flux both decrease, the $(\alpha, n)$ neutron flux increases.

Analysis of ORIGEN-S data shows that at lower cooling times the majority of neutrons are produced from spontaneous fission of heavy nuclides with ${ }^{244} \mathrm{Cm}$ as the largest contributor. However, the increase in $(\alpha, n)$ reactions with increasing cooling times is due to the increase in ${ }^{241} \mathrm{Am}$ which decays from ${ }^{241} \mathrm{Pu}$. As shown in Figure 4, ${ }^{241} \mathrm{Am}$ is the major contributor to the $(\alpha, \mathrm{n})$ reaction neutron source term is spent fuel.

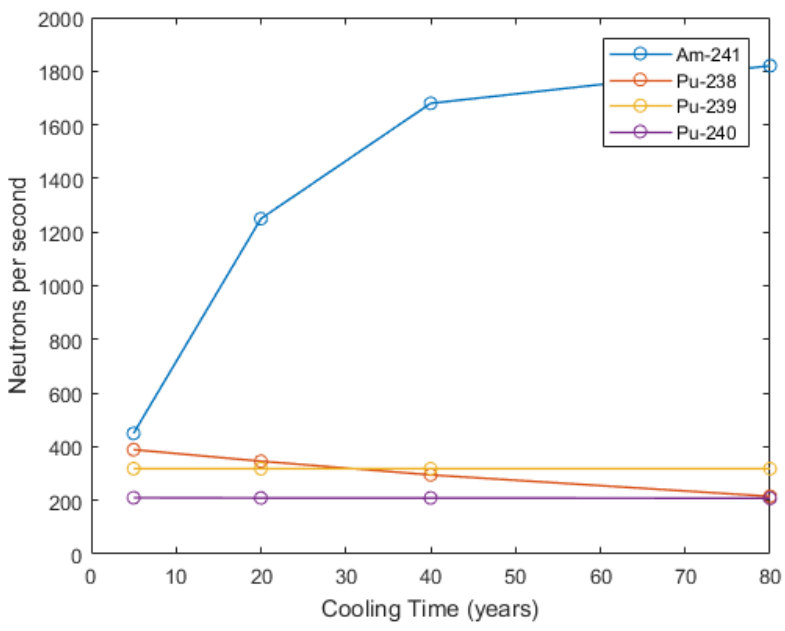

Fig. 4. Change in $(\alpha, n)$ reaction rate by $\alpha$ emitter as a function of cooling time. The increase in alpha emissions from ${ }^{241} \mathrm{Am}$ results in the increase in $(\alpha, n)$ reactions.

Separating spontaneous fission neutrons and $(\alpha, n)$ reaction neutrons in computational models is straightforward. A detector in the field would only be able to measure neutron counts and energies. Therefore, it was necessary to create a method to separate these two neutron sources from a detector measurement.

Based on the characteristics of their spectra, the spontaneous fission neutrons and $(\alpha, n)$ reaction neutrons can be estimated from a total neutron spectrum. The spontaneous fission neutrons peak between 0.8 to $1 \mathrm{MeV}$ and have a long tail extending to 12 to $15 \mathrm{MeV}$. The spectrum for $(\alpha, n)$ reaction neutrons has a softer curve with a maximum between 2.5 and $3.0 \mathrm{MeV}$. The $(\alpha, n)$ neutron spectra tail ends at approximately $5 \mathrm{MeV}$.

Using these characteristics, the results are separated into two integrals. The first is from 0 to $1.6 \mathrm{MeV}$, covering most of the spontaneous fission neutrons. The second is from $1.6 \mathrm{MeV}$ to $3.6 \mathrm{MeV}$, spanning most of the $(\alpha, n)$ reaction neutrons. Figure 5 shows the characteristic ranges on a graph of the total neutron spectrum for a spent fuel rod with 4 percent initial enrichment, $15 \mathrm{GWd} / \mathrm{MTU}$ burnup and a 40-year cooling time.

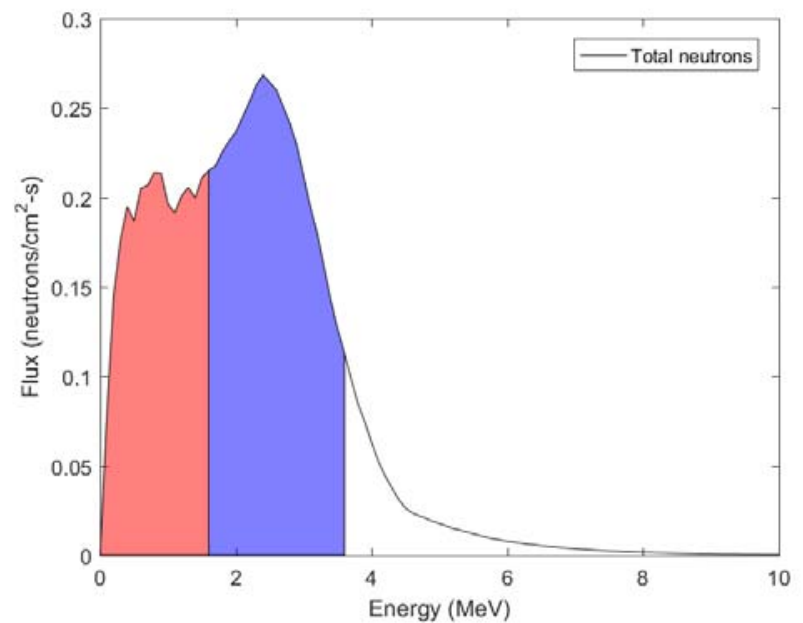

Fig. 5. Characteristic energy ranges for spontaneous fission and $(\alpha, n)$ neutrons. The range on the left from 0 to $1.6 \mathrm{MeV}$ is for spontaneous fission neutrons and the range on the right from $1.6 \mathrm{MeV}$ to $3.6 \mathrm{MeV}$ is for $(\alpha, n)$ neutrons.

Figure 6 shows the energy regions of interest for spontaneous fission and $(\alpha, n)$ reactions plotted versus cooling time. As cooling time increases, the spontaneous fission neutron flux decreases at a faster rate than the $(\alpha, n)$ reaction neutron flux.

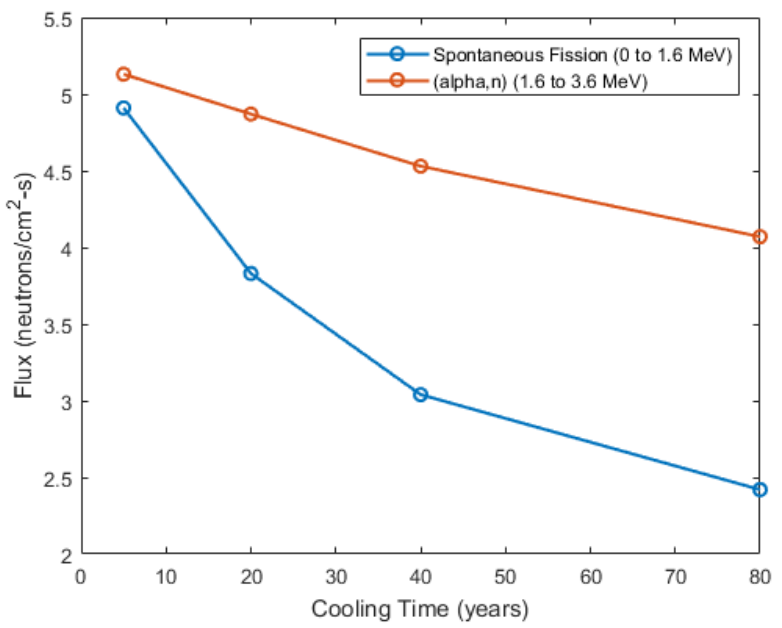

Fig. 6. Change in neutron flux in characteristic energy ranges as a function of cooling time. The spontaneous fission flux decreases at a faster rate, allowing characterizing the cooling time based on the ratio of the two fluxes. 
Identification of this trend enables characterizing the cooling time of spent nuclear fuel in dry storage casks. Further refinements to the methodology will be necessary to increase the accuracy of the cooling time prediction.

\section{CONCLUSIONS}

The linkage code developed for ORIGEN-S and MCNP6 allows analysis of transported neutron source terms for spent fuel rods and assemblies from the NGSI Spent Fuel Libraries. ORIGEN-S calculates the neutron emissions from the spent fuel rods. MCNP6 performs the transport and shielding calculations.

The identification of neutron spectral characteristics in individual rods and 17X17 PWR assemblies are applicable to spent fuel assemblies in dry cask storage. The first characteristic identified is the varying ratio of spontaneous fission neutrons versus $(\alpha, n)$ reactions over time. This is due to the long term increase in ${ }^{241} \mathrm{Am}$, a significant $\alpha$ source.

This method offers advances over the method of fingerprinting casks. Neutron source spectral characteristics allows restoration of continuity of knowledge, as it is able to determine parameters of spent fuel inside dry storage casks. The next step of this work is examining other trends in the total neutron signal that correlate with spent fuel parameters.

\section{REFERENCES}

[1] M. Tarvainen, F. Levai, T. E. Valentine, M. Abhold, and B. Moran, "NDA techniques for spent fuel verification and radiation monitoring," 1997.

[2] E. Rauch, "Developing a Method for Unique Identification of Used Fuel Storage Casks Using Neutrons," 2014.

[3] K. P. Ziock, P. Vanier, L. Forman, G. Caffrey, and A. Lebrun, "The Feasibility of Cask 'Fingerprinting' as a Spent-Fuel, Dry-Storage Cask Safeguards Technique," 2005.

[4] A. Rimpler, "Bonner sphere neutron spectrometry at spent fuel casks," Nucl. Instruments Methods Phys. Res. Sect. A Accel. Spectrometers, Detect. Assoc. Equip., vol. 476, no. 1-2, pp. 468-473, 2002.

[5] R. A. Weldon, M. L. Fensin, and H. R. Trellue, "Total neutron emission generation and characterization for a Next Generation Safeguards Initiative spent fuel library," Prog. Nucl. Energy, vol. 80, pp. 45-73, 2015.

[6] J. D. Galloway, H. R. Trellue, M. L. Fensin, and B. L. Broadhead, "Design and Description of the NGSI Spent Fuel Library with Emphasis on the Passive Gamma Signal," J. Nucl. Mater. Manag., vol. XL, no. 3, pp. 25-35, 2012.

[7] I. C. Gauld, S. M. Bowman, B. D. Murphy, and P. Schwalbach, "Applications of ORIGEN to Spent Fuel Safeguards and Non-Proliferation," 2015.

[8] M. D. DeHart and O. W. Hermann, "An Extension of the Validation of SCALE (SAS2H) Isotopic Predictions for PWR Spent Fuel," 1996.

[9] B. C. Kiedrowski, F. B. Brown, L. J. Cox, N. A. Gibson, A. S. Bennett, and M. A. Gonzales, "MCNP6 Shielding Vaidation Suite: Past, Present, and Future," vol. $836,2011$.
[10] M. B. Chadwick, P. Oblozinski, M. Herman, N. M. Greene, and R. D. McKnight, "ENDF/B-VII.0: Next Generation Evaluated Nuclear Data Library for Nuclear Science and Technology," vol. 107, no. 12, pp. 2931-3060, 2006. 